\title{
Going in circles: conserved mechanisms control radial patterning in the urinary and digestive tracts
}

\author{
Cathy Mendelsohn
}

Departments of Urology and Pathology, Columbia University, New York, New York, USA.

\begin{abstract}
Radial patterning in the urinary tract and gut depends on reciprocal signaling between epithelial cells, which form mucosa, and mesenchyme, which forms smooth muscle and connective tissue. These interactions depend on sonic hedgehog (Shh), which is secreted by epithelial cells and induces expression of bone morphogenetic protein 4 (Bmp4), a signaling molecule required for differentiation of smooth muscle progenitors. Patterning of the specialized mucosa lining the anterior-posterior (A-P) axis may be controlled independently by regionally expressed mesenchymal transcription factors. A study by Airik et al. in this issue of the JCI reveals that T-box 18 (Tbx18), a transcription factor selectively expressed in ureteral mesenchyme, regulates smooth muscle differentiation by maintaining Shh1 responsiveness in mesenchymal progenitors (see the related article beginning on page 663). Deletion of $T b x 18$ resulted in defective urothelial differentiation at the level of the ureter, suggesting that $T b x 18$ acts via mesenchyme as an important regulator of A-P patterning in the urinary tract.
\end{abstract}

Efficient urine transport is crucial for renal function, preventing damage induced by buildup of urine in the kidney. Urinary tract defects that impede urine transport occur in $1-2 \%$ of the human population and can cause obstruction, renal scarring, and end-stage renal disease (1-3). Urine is normally transported from the kidney to the bladder via the renal pelvis and ureters, which are lined with a water-resistant transitional epithelium, the urothelium, and are surrounded by a muscular coat that mediates myogenic peristalsis (Figure 1).

The kidneys and ureters are derived from the ureteric bud, a posterior branch of the Wolffian ducts, paired epithelial tubes that at late developmental stages differentiate into the vas deferens, seminal vesicles, and epididymis in males and regress in females. The ureteric bud tip invades kidney mesenchyme and undergoes successive rounds of branching morphogenesis that give rise to the renal collecting duct system. The posterior ureteric bud stalk differentiates into the ureter, a muscular tube that conducts urine from the kidney to the bladder (Figure 1). The bladder and urethra form from

Nonstandard abbreviations used: A-P, anteriorposterior; Barx1, BarH-like homeobox 1; Bmp4, bone morphogenetic protein 4; Shh, sonic hedgehog; Tbx18, T-box 18; Upk, uroplakin.

Conflict of interest: The author has declared that no conflict of interest exists.

Citation for this article: J. Clin. Invest. 116:635-637 (2006). doi:10.1172/JCI27985. the cloaca, an endodermal pouch that is partitioned into the hindgut dorsally and the urogenital sinus ventrally. Initially the ureters drain into the Wolffian ducts and hence are not connected directly with the bladder. Formation of mature connections occurs prior to the onset of renal function, when the ureter orifice is transposed from the Wolffian duct to the urogenital sinus. Subsequent growth and expansion of the bladder then shifts the position of the ureter orifice anterior to its final insertion site in the bladder neck.

\section{Mouse models of physical obstruction}

Recently, mouse models have been generated that display various types of obstruction, enabling us to begin to understand how urinary tract defects occur in humans. For example, deletion of the genes Spronty1, Slit2, or Foxc1/Foxc2 or maternal exposure to retinoic acid results in obstruction due to formation of supernumerary ureters that join the lower urinary tract abnormally (4-7). The configuration of these duplicated systems is typical to that in humans: Ureters that emerge at the proper site on the Wolffian duct close to the urogenital sinus generally undergo normal transposition, joining the bladder neck, while branches that form at abnormally high positions on the Wolffian duct fail to undergo transposition, joining the lower urinary tract outside the normal insertion site, for example in the genital tract or urethra (ref. 8; reviewed in ref. 9). Once renal function begins, "plumbing" problems linked to malpositioned ureters can disrupt urine transport and cause severe injury to the kidney. Proper differentiation of the specialized cell types that line the ureter is also crucial for efficient urine transport. Abnormalities in urothelial differentiation can cause physical or functional obstruction. Ureteral valves, an example of physical obstruction, are associated with overgrowth of the transitional epithelium that occludes the ureteral lumen, blocking urine transport (10). A similar phenotype was observed in mouse mutants lacking uroplakins, a family of proteins that form the hexagonal plaques lining the apical surface of the urothelium (11). Deletion of uroplakin 2 (Upk2) or Upk3 results in hyperplasia of the ureteral epithelium that effectively blocks urine flow, resulting in obstruction and, in some cases, renal failure $(12,13)$.

\section{Mouse models of functional obstruction}

Functional obstruction - defective urine transport in the absence of physical blockage - may be linked to defects in the ureter smooth muscle coat that interfere with peristalsis or result in inadequate structural integrity $(14,15)$. Recent studies suggest that ureteral smooth muscle differentiation depends on sonic hedgehog (Shb) and bone morphogenetic protein 4 (Bmp4) $(14,16,17)$. Conditional inactivation of Shb in urinary tract epithelia results in renal hypoplasia, hydroureter, and hydronephrosis. Hydronephrosis and hydroureter were likely to be due to defective differentiation of periureteral mesenchyme (14). These studies further suggest that $S h b$ acts in part by regulating proliferation of mesenchymal smooth muscle progenitors and in part by inducing expression of Bmp4 in ureteral mesenchyme, which is necessary for recruitment of periureteral mesenchyme and for its differentiation into smooth muscle $(16,17)$. A similar Shb-and Bmp4-dependent pathway operates in the gut, establishing basic epithelial and mesenchymal patterning along the radial axis $(18,19)$. 
A

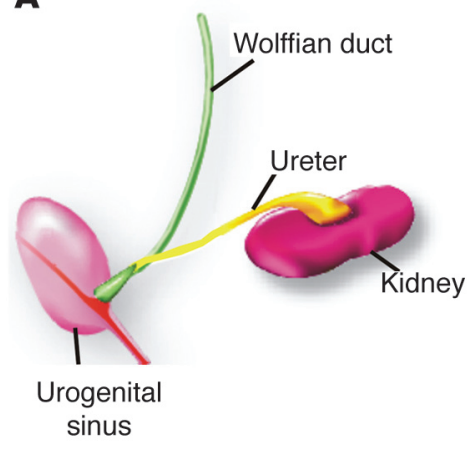

B

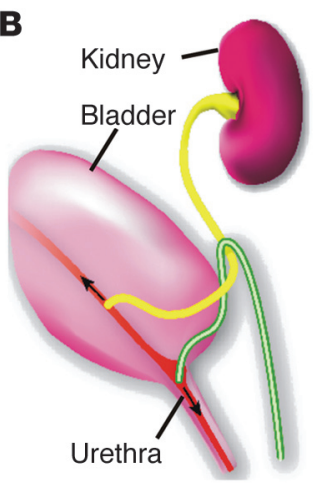

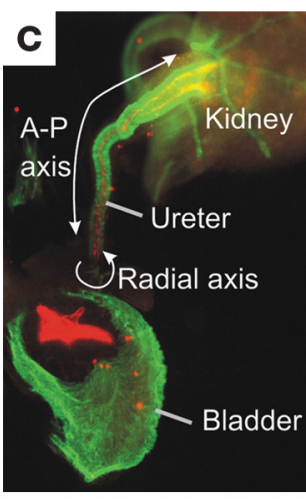

Urothelium $\alpha-S M A$
Tbx18, a mesenchymal transcription factor that controls radial and anterior-posterior patterning in the urinary tract

A study in this issue of the JCI by Airik et al. (20) suggests that T-box 18 (Tbx18), a T-box transcription factor selectively expressed in ureteral mesenchyme, is important for both radial and anterior-posterior (A-P) patterning, inducing terminal differentiation of the ureteral epithelium. Mouse mutants lacking $T b x 18$ displayed hydronephrosis and hydroureter without physical blockage and are hence to my knowledge a new model of functional obstruction. Histological analysis of mutant ureters reveals defects in both muscle and urothelial differentiation. In wild-type embryos, ureteral mesenchyme aggregated around the ureter and differentiated into the circular and longitudinal smooth muscle coat. Deletion of $T b \times 18$ resulted in deficiency of smooth muscle; ureteral mesenchyme failed to condense around the ureter and was instead mislocalized to the surface of the kidney, forming a fibrous outer layer. Analysis of gene expression revealed that 2 Shb targets, Bmp 4 and Patched 1 , were downregulated in the mesenchyme of $T b \times 18$ mutants, suggesting that $T b x 18$ is likely to be important for controlling the response to hedgehog signaling in mesenchyme and for expression of Bmp4, an important mediator of smooth muscle differentiation (Figure 2A). Establishment of radial patterning in the gut is also controlled by an $S h b$ - and Bmp4-dependent signaling pathway; however, further differentiation of specialized epithelia that line the A-P axis probably depends on regionally expressed mesenchymal transcription factors. For example, differentiation of the mucosal lining of the stomach requires BarH-like homeobox 1 (Barx1), a transcription factor that is selectively expressed in stomach mesenchyme (21).

Differentiation of epithelia lining the renal collecting ducts, bladder, and urethra was apparently normal in Tbx18 mutants; however, differentiation of the mucosa lining the ureter was blocked, the ureteral epithelium remained undifferentiated (20), and uroplakins, markers of urothelial differentiation (22), were not expressed (20). The observation that expression of the Wnt inhibitor Sfrp2 was downregulated in mutant mesenchyme raises the intriguing possibility that $T b \times 18$ may act in a manner similar to Barx 1 in the stomach, inducing expression of signals that control urothelial patterning

\section{Figure 1}

The embryonic urinary tract. (A and B) Schematic representations of the mouse urinary tract at E11.5 (A) and E15 (B). (C) Wholemount urinary tract from an E15 mouse stained with uroplakin (red), a marker of the urothelium, and $\alpha$-SMA (green), a marker of smooth muscle. The A-P and radial axes are shown with white arrows. Note that urothelial differentiation occurs in renal pelvis, ureters, and bladder, but not in the kidney, which is lined with a distinct mucosa. Magnification, $\times 10$. by suppressing Wnt signaling (Figure 2B). Whether this is or is not the case awaits further investigation to define the role of Wnt signaling during ureter differentiation and the requirement for Sfrp 2 in urothelial morphogenesis. That epithelial differentiation in the urinary tract is controlled by signals from mesenchyme is well established; however, the identity of these mesenchymal signals has been unknown (23). The studies reported here by Airik et al. (20) suggest that Tbx18 may be such a mesenchymal signal, controlling ureter differentiation. Whether other transcription factors (or even Tbx family members) control patterning of specialized epithelia at other sites in the urinary tract is an interesting possibility.

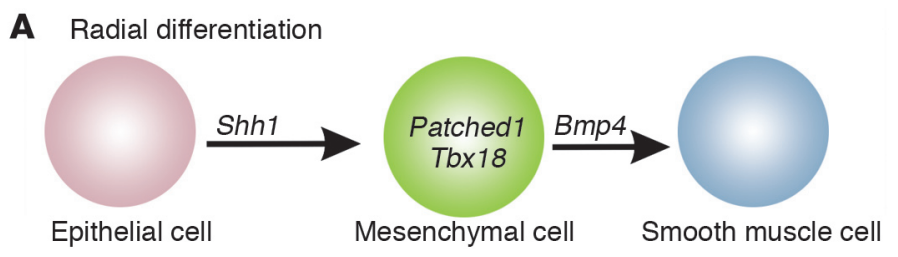

B Urothelial differentiation

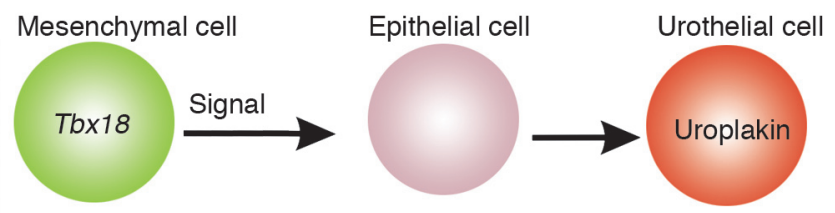

Figure 2

A model of $T b \times 18$ action in radial patterning and urothelial differentiation. (A) Tbx18 is selectively expressed in ureteral mesenchyme, where it is required for smooth muscle differentiation and for differentiation of the urothelium, the specialized mucosa that provides barrier function. Tbx18 controls smooth muscle differentiation by regulating Shh responsiveness in subepithelial mesenchyme (3). Shh secreted from ureteral epithelia induces expression of Patched1 and $B m p 4$, a signaling molecule critical for recruitment of ureteral mesenchyme and for its differentiation into smooth muscle. (B) Tbx18 is selectively expressed in ureteral mesenchyme and is important for differentiation of the urothelium lining the ureters, but not the urothelium lining the bladder and urethra. 
Address correspondence to: Cathy Mendelsohn, Departments of Urology and Pathology, Columbia University, 650 West 168th Street, New York, New York 10032, USA. Phone: (212) 305-1591; Fax: (212) 305-6851; E-mail: clm20@columbia.edu.

1. Chevalier, R.L. 2004. Obstructive nephropathy and the developing kidney: too little or too much angiotensin? Kidney Int. 65:1517-1518.

2. Chevalier, R.L. 1998. Pathophysiology of obstructive nephropathy in the newborn. Semin. Nephrol. 18:585-593.

3. Scott, J.E., and Renwick, M. 1988. Antenatal diagnosis of congenital abnormalities in the urinary tract. Results from the Northern Region Fetal Abnormality Survey. Br. J. Urol. 62:295-300.

4. Kume, T., Deng, K., and Hogan, B.L. 2000. Murine forkhead/winged helix genes Foxc1 (Mf1) and Foxc2 (Mfh1) are required for the early organogenesis of the kidney and urinary tract. Development. 127:1387-1395.

5. Grieshammer, U., et al. 2004. SLIT2-mediated $\mathrm{ROBO} 2$ signaling restricts kidney induction to a single site. Dev. Cell. 6:709-717.

6. Basson, M.A., et al. 2005. Sprouty1 is a critical regulator of GDNF/RET-mediated kidney induction. Dev. Cell. 8:229-239.

7. Batourina, E., et al. 2005. Apoptosis induced by vitamin A signaling is crucial for connecting the ureters to the bladder. Nat. Genet. 37:1082-1089.

8. Mackie, G.G., and Stephens, F.D. 1975. Duplex kidneys: a correlation of renal dysplasia with position of the ureteral orifice. J. Urol. 114:274-280.

9. Ichikawa, I., Kuwayama, F., Pope, J.C., Stephens, F.D., and Miyazaki, Y. 2002. Paradigm shift from classic anatomic theories to contemporary cell biological views of CAKUT. Kidney Int. 61:889-898.

10. Wall, B., and Wachter, E. 1952. Congenital ureteral valve: its role as a primary obstructive lesion: classification of the literature and report of an authentic case. J. Urol. 68:684-690.

11. Wu, X.R., et al. 1994. Mammalian uroplakins. A group of highly conserved urothelial differentiation-related membrane proteins. J. Biol. Chem. 269:13716-13724.

12. Kong, X.T., et al. 2004. Roles of uroplakins in plaque formation, umbrella cell enlargement, and urinary tract diseases. J. Cell Biol. 167:1195-1204.

13. Hu, P., et al. 2001. Ablation of uroplakin III gene results in small urothelial plaques, urothelial leakage, and vesicoureteral reflux. Urology. 57:117.

14. Yu, J., Carroll, T.J., and McMahon, A.P. 2002. Sonic hedgehog regulates proliferation and differentiation of mesenchymal cells in the mouse metanephric kidney. Development. 129:5301-5312.

15. Chang, C.P., et al. 2004. Calcineurin is required in urinary tract mesenchyme for the development of the pyeloureteral peristaltic machinery. J. Clin. Invest. 113:1051-1058. doi:10.1172/JCI200420049.

16. Miyazaki, Y., Oshima, K., Fogo, A., Hogan, B.L., and
Ichikawa, I. 2000. Bone morphogenetic protein 4 regulates the budding site and elongation of the mouse ureter. J. Clin. Invest. 105:863-873.

17. Raatikainen-Ahokas, A., Hytonen, M., Tenhunen, A., Sainio, K., and Sariola, H. 2000. BMP-4 affects the differentiation of metanephric mesenchyme and reveals an early anterior-posterior axis of the embryonic kidney. Dev. Dyn. 217:146-158.

18. Sukegawa, A., et al. 2000. The concentric structure of the developing gut is regulated by Sonic hedgehog derived from endodermal epithelium. Development. 127:1971-1980.

19. Roberts, D.J., Smith, D.M., Goff, D.J., and Tabin, C.J. 1998. Epithelial-mesenchymal signaling during the regionalization of the chick gut. Development. 125:2791-2801

20. Airik, R., Bussen, M., Singh, M.K., Petry, M., and Kispert, A. 2006. Tbx18 regulates the development of the ureteral mesenchyme. J. Clin. Invest. 116:663-674. doi:10.1172/JCI26027.

21. Kim, B.M., Buchner, G., Miletich, I., Sharpe, P.T., and Shivdasani, R.A. 2005. The stomach mesenchymal transcription factor Barx1 specifies gastric epithelial identity through inhibition of transient Wnt signaling. Dev. Cell. 8:611-622.

22. Liang, F.X., et al. 2005. Cellular basis of urothelial squamous metaplasia: roles of lineage heterogeneity and cell replacement. J. Cell Biol. 171:835-844.

23. Lipschutz, J.H., Young, P., Taguchi, O., and Cunha, G.R. 1996. Urothelial transformation into functional glandular tissue in situ by instructive mesenchymal induction. Kidney Int. 49:59-66.

\title{
Ghrelin receptor mutations — too little height and too much hunger
}

\author{
Birgitte Holst and Thue W. Schwartz
}

Laboratory for Molecular Pharmacology, Panum Institute, University of Copenhagen, Copenhagen, Denmark.

\begin{abstract}
The ghrelin receptor is known from in vitro studies to signal in the absence of the hormone ghrelin at almost $50 \%$ of its maximal capacity. But, as for many other 7-transmembrane receptors, the in vivo importance of this ligand-independent signaling has remained unclear. In this issue of the JCI, Pantel et al. find that a natural mutation in the ghrelin receptor, Ala204Glu, which is associated with a selective loss of constitutive activity without affecting ghrelin affinity, potency, or efficacy, segregates in $\mathbf{2}$ families with the development of short stature (see the related article beginning on page 760). By combination of the observations from this study with those related to the phenotype of subjects carrying another natural ghrelin receptor mutation, Phe279Leu, having identical molecular-pharmacological properties, it is proposed that selective lack of ghrelin receptor constitutive signaling leads to a syndrome characterized not only by short stature, but also by obesity that apparently develops during puberty.
\end{abstract}

Seven-transmembrane segment (7TM) or $\mathrm{G}$ protein-coupled receptors can signal without any agonist present (1). This

Nonstandard abbreviations used: CB1, cannabinoid type $1 ; \mathrm{GH}$, growth hormone; 7TM, 7-transmembrane segment.

Conflict of interest: The authors have declared that no conflict of interest exists.

Citation for this article: J. Clin. Invest. 116:637-641 (2006). doi:10.1172/JCI27999. phenomenon is a simple reflection of the molecular activation mechanism where the receptor is in an equilibrium between inactive and active conformations and can relatively easily access the active conformation without the presence of an agonist (2). The degree of ligand-independent or constitutive signaling varies among receptors but in most cases only represents a small fraction of the maximal signaling capacity and is consequently generally ignored. However, among receptors involved in the control of appetite and energy expenditure, such constitutive activity appears to have important functional consequences. The cannabinoid type 1 (CB1) receptor, which is the target for the novel antiobesity drug rimonabant - acting as a combined antagonist and inverse agonist - and the ghrelin receptor both signal with around 50\% activity in the absence of ligand (3-5). Nevertheless, the physiological importance of such constitutive signaling has been very hard to establish in vivo, mainly because of the lack of appropriate pharmacological tools. Inverse agonists, i.e., ligands that inhibit the constitutive receptor signaling, in most cases also act as antagonists, i.e., they also block the action of the endogenous agonist. Thus, it is very hard in the in vivo setting to differentiate between an effect on constitutive receptor signaling and a blocking effect on receptor access to an endogenous ligand. In this issue of 